\title{
EFFECT OF NITROGEN AND POTASSIUM LEVELS ON YIELD AND YIELD COMPONENTS OF SOME RICE GENOTYPES UNDER SALIN SOILS. \\ Hagrus, A. M. ${ }^{1}$; B. A. Zayed ${ }^{2}$; N. F. Doula ${ }^{1}$ and A. M. Zayed ${ }^{2}$ \\ 1 Agronomy department, Fac. of Agric., Al-Zahra Univ., Egypt. \\ 2Rice Res. and Training Center, Sakha, Field Crop Res., Agric. Res. Center, Giza, Egypt
}

\begin{abstract}
Integrating rice crop management involving using salt tolerant variety and proper cultivation is the main strategy to increase rice production under saline soils.

Two field Experiments were conducted during 2006 and 2007 Rice growing seasons at the research farm of El-Sirw Agricultural Research Station Damietta Governorate, Egypt. The experiments were conducted to study the effect of three nitrogen levels 0,35 and70 $\mathrm{kg} \mathrm{N} / \mathrm{fed}$ and three potassium levels $0,24,48 . \mathrm{kgK} 2 \mathrm{O} / \mathrm{fed}$ and their interaction on growth yield and its components of some rice genotypes (SK2046H or EHR1) (SK2034H or EHR2) i.e. hybrid ones and Giza 178 i.e. inbred one under saline soil conditions. Split - split plot design with four replications was used in the experiments, the varieties were distributed in the main plots the sub- plots were allocated to three nitrogen rates the sub plot were devoted to three potassium rates.

The main obtained results could be summarized. The nitrogen levels had positive and significant effects on plant height, tiller number, panicle length, panicle number, filled grain / panicle, panicle weight, 1000 - grain weight, grain yield and straw yield. All rice cultivar significantly responded to nitrogen fertilizer up to $70 \mathrm{~kg}$ $\mathrm{N} / \mathrm{fed}$. Potassium fertilizer significantly increased rice growth, yield components and rice grain yield in both seasons. Rice response to potassium fertilizer was significantly up to higher potassium level of $48 \mathrm{~kg} \mathrm{K2O/fed.} \mathrm{Egyptian} \mathrm{hybrid} \mathrm{one} \mathrm{EHR1} \mathrm{(SK2034H)}$ with $70 \mathrm{kgN} / \mathrm{fed}$ and $48 \mathrm{kgK} 2 \mathrm{O} / \mathrm{fed}$ gave the highest values of estimated growth parameters, yield components and grain yield under current study. Rice genotypes significantly varied in their growth traits, yield component and grain yield as well as harvest index. (SK2034H) hybrid rice variety significantly surpassed other two tested genotypes followed by Giza 178 rice variety. (SK2046H) occupied the last rank.

It could be concluded that EHR 1 had to be fertilized by $70 \mathrm{~kg} \mathrm{~N} / \mathrm{fed}$ and $48 \mathrm{~kg}$ $\mathrm{k}_{2} \mathrm{O} / \mathrm{fed}$ under current saline conditions for producing considerable grain yield.
\end{abstract}

\section{INTRODUCTION}

Rice (Oryza sativa, $L$ ) is considered as one of the most important food cereal grain crops after wheat in Egypt. It is a staple food for nearly one half of the world population most of them live in developing countries In the 2010 year the annual area cultivated by rice in Egypt is about 1.5 million feddans which is about 0.63 million hectares and the total rice production reached about 6 million tons with a national average of 9.30 ton $/ \mathrm{ha}$.

Rising rice productivity under saline soil is very important to increase rice average yield since rice is growing under wide salt affected area with low yield. 
Hagrus, A. M. et al.

Exploring hybrid rice under saline using its high heterosis might increase rice yield under such condition in which Zayed et al.,(2006b,2007 and 2010) found great variation between hybrid rice and inbred rice under saline. Moreover, Gautam.A.K (2004) and Abou Khalifa (20005) stated variation between hybrid and inbred rice related to their growth, yield components and rice grain yiled

Nitrogen fertilization is applied to meet the needs of the crop during the early growth stages accumulated in the vegetative parts to be utilized for grain formation for ensuring economic grain yield. The large portion of the nitrogen is absorbed during differentiation stage where the leaves and stems contain a large portion of the nitrogen uptake by the plant. Also, nitrogen fertilization application plays a vital role in nitrogen \% in rice grain and nitrogen uptake by rice plant (Ebaid and Ghanem 2001).

El-Shayeb (2003) found that increasing nitrogen up to $80 \mathrm{kgN} / \mathrm{fed}$ significantly increased plant height, panicle length, panicle number, filled grains / panicle, panicle weight, straw and grain yield. Zayed et al. (2006b) and Lin et al. (2009) stated that increasing nitrogen rate up to $180 \mathrm{~kg} \mathrm{~N} / \mathrm{ha}$ significantly increased rice growth, yield and yield components under saline soil. Bahmanyar and Soodaee (2010) reported that increasing nitrogen levels significantly increased plant height, leaf area, yield components and grain yield.

Potassium recommended to be applied under saline soil for alleviation salinity tolerance and rice productivity. Potassium fertilization had a marked influence on the over all nutrient levels in grain. Nour et al. (1997) and Ebaid and Ghanem (2001) found that potassium fertilization increased rice yield and its components. Under saline soil, Zayed (2002), Shahzada et al.( 2007), Zayed et al. (2007) and Bahmanyar and Soodaee (2010) studied the effect of three potassium levels $(0,20$ and $40 \mathrm{~kg} \mathrm{~K} 2 \mathrm{O} / \mathrm{fed})$ on the yield components and chemical contents of rice. They found that potassium application had positive effects on growth, yield components, grain yield and nitrogen.

Zayed et al. (2006b and 2007) reported significant differences in rice hybrid and inbred regarding their response to both nitrogen and potassium fertilizer rates.

The current study amid to find out the optimum nitrogen and potassium rate for both hybrid and inbred rice varieties under saline soils.

\section{MATERAILS AND METHODS}

Two field Experiments were conducted during 2006 and 2007 seasons at the Research Farm of El-Sirw Agricultural Research Station, Damietta province, Egypt. The experiments were amid to study the effect of three nitrogen levels i.e. 0, 35and $70 \mathrm{~kg} \mathrm{~N} / \mathrm{fed}$, and three potassium levels i.e. 0 , 24and $48 \mathrm{kgK} 2 \mathrm{O} / \mathrm{fed}$ as well as their interaction on growth, yield and its components of some rice genotypes (SK2046H or EHR1), (SK2034H or EHR2) as hybrid rice and Giza 178 as inbred rice under saline soil conditions. A split - split plot design with four replications was used, the varieties were 
distributed in the main and plot the sub- plots were allocated to the three nitrogen rates while, the sub-sub plot were devoted to the three potassium rates.

Seedling of 30 days old of rice was transplanted with 3 seedlings hill-1, spaced at $20 \times 20 \mathrm{~cm}$. Transplanting was done on April, $20^{\text {th }}$ and harvested on September $1^{\text {st }}$. Nitrogen fertilizer in the abovementioned rates was imposed in 4 equal doses i.e. 15 days after transplanting (DAT), mid-tillering stage, panicle initiation stage, and end of booting stage as recommended under saline soil. All plots received $90 \mathrm{~kg} \mathrm{P} \mathrm{O}_{5} /$ ha in the form of calcium super phosphate. Plot area was adjusted to $10 \mathrm{~m}^{2}$. The soil was clay and the soil chemical properties were listed in Table 1.

Table 1: Soil chemical properties at the experimental sites during 2010 and 2011seasons.

\begin{tabular}{|c|c|c|c|c|c|c|c|c|}
\hline \multirow{2}{*}{ season } & \multirow{2}{*}{ pH } & \multirow{2}{*}{$\begin{array}{c}\mathrm{EC} \\
\mathrm{dS} \mathrm{m}^{-1}\end{array}$} & \multicolumn{3}{|c|}{ Cation meq $\mathrm{L}^{-1}$} & \multicolumn{3}{|c|}{ Anion Meq $\mathrm{L}^{-1}$} \\
\hline & & & $\mathrm{Ca}^{++}+\mathrm{Mg}^{++}$ & $\mathrm{Na}^{+}$ & $\mathbf{K}^{+}$ & $\mathrm{SO}{ }_{4}$ & $\mathrm{Cl}^{-}$ & $\mathrm{HCO}_{3}^{-}$ \\
\hline 2006 & 8.3 & 7.5 & 20.0 & 58.0 & 0.32 & 26.5 & 45.3 & 8.0 \\
\hline 2007 & 8.2 & 6.5 & 18.0 & 57.0 & 0.31 & 20.0 & 46.0 & 7.0 \\
\hline \multicolumn{9}{|c|}{ Available nutrients $\mathbf{~ m g ~ k g}^{-1}$} \\
\hline & $\mathbf{N}$ & $\mathbf{P}$ & $\mathbf{K}$ & $\mathrm{Zn}$ & $\mathbf{S}$ & & $\mathrm{Fe}$ & Cu \\
\hline 2006 & 28.0 & 9.12 & 245.0 & 1.22 & 10.7 & & 5.00 & 6.2 \\
\hline 2007 & 26.0 & 9.35 & 280.0 & 1.16 & 10.5 & & 5.13 & 6.0 \\
\hline
\end{tabular}

At heading stages, ten hills from each plot were taken to estimate leaf area index (LAl) and dry mater (the dry samples were weighed and dry matter $\mathrm{g}$ $\mathrm{m}^{-2}$ was computed.

At harvest, panicles of ten guarded hills for each plot were counted to determine the number of panicles $\mathrm{m}^{-2}$ and also plant height $(\mathrm{cm})$ was measured. Ten main panicles from each subplot were packed to determine panicle length $(\mathrm{cm})$, filled and unfilled grains panicle ${ }^{-1}$, panicle and 1000-grain weights $(\mathrm{g})$. The plants of the six inner rows of each sub-sub plot were harvested, dried, threshed, then grain and straw yields were determined at 14 $\%$ moisture content and converted into tha-1. Soil was sampled before planting for soil chemical analysis according to Piper, 1950.

All data collected were subjected to standard statistical analysis following the proceeding described by Gomez and Gomez (1984) using the computer program (IRRISTAT). The treatment means were compared using Duncan's multiple range test (Duncan, 1955). Indicate the significant at $5 \%$ and $1 \%$ levels of probability, respectively, while NS means not significant.

\section{RESULTS AND DISCUSSION}

\section{Varietals differences:}

Data in Tables2,3and4 showed that the tested three rice genotypes, hybrid ones; EHR1, EHR2 as well as inbred one, Giza 178 significantly varied The measured traits; dry matter, leaf area index (LAI), heading date, tillers number $/ \mathrm{m}^{2}$, panicle numbers $/ \mathrm{m}^{2}$, plant height, panicle length, filled 
grains /panicle, unfilled grains/panicle, panicle weight, 1000-grain weight, grain yield (t/fed), straw yield (t/fed) and harvest index were significantly differed in both seasons except, filled grain /panicle in 2006 season. EHR1 hybrid rice variety showed its superiority in the above-mentioned traits in both seasons followed by EHR2. Giza 178 inbred rice variety was in the last rank regarding the abovementioned traits in both seasons. The superiority of hybrid rice variety was mainly due to their higher heterosis than that inbred one. Similar findings had been reported by Gautam et al. (2004), Abou Khalifa (2005), Zayed et al. (2005), Zayed et al. (2006a and b) Zayed et al (2010).

Table (2): Dry matter(g) $/ \mathrm{m}^{2}$ leaf area index ,day to heading, plant height and number of tiller $/ \mathrm{m}^{2}$ of some rice genotypes as affected by nitrogen and potassium level under saline soil during 2006 and 2007 seasons

\begin{tabular}{|c|c|c|c|c|c|c|c|c|c|c|}
\hline \multirow{2}{*}{$\begin{array}{l}\text { Characters } \\
\text { Treatments }\end{array}$} & \multicolumn{2}{|c|}{ Dry matter $\mathbf{g} / \mathrm{m}^{2}$} & \multicolumn{2}{|c|}{$\begin{array}{l}\text { Leaf area } \\
\text { index }\end{array}$} & \multicolumn{2}{|c|}{$\begin{array}{l}\text { Days to } \\
\text { heading }\end{array}$} & \multicolumn{2}{|c|}{$\begin{array}{r}\text { Plant height } \\
\text { / plant }(\mathbf{c m})\end{array}$} & \multicolumn{2}{|c|}{ No of tillers $/ \mathrm{m}^{2}$} \\
\hline & 2006 & 2007 & 2006 & 2007 & 2006 & 2007 & 2006 & 2007 & 2006 & 2007 \\
\hline Hybrid 1 & 1294.7 & 1274.1 & 4.02 & 4.05 & 108.3 & 108.6 & 90.02 & 96.50 & 472.00 & 402.58 \\
\hline Hybrid2 & 1277.9 & 1254.5 & 3.82 & 3.86 & 108.3 & 107.59 & 85.03 & 95.66 & 431.00 & 396.31 \\
\hline Giza178 & 1217.3 & 1197.9 & 3.67 & 2.86 & 107.1 & 106.8 & 83.45 & 93.79 & 421.00 & 347.38 \\
\hline LSD 0.05 & 0.45 & 0.53 & 0.26 & 0.07 & 0.26 & 0.21 & 1.73 & 1.31 & 0.57 & 0.33 \\
\hline \multicolumn{11}{|l|}{$\mathrm{N}$ levels } \\
\hline Okg/fed & 1044.4 & 1012.7 & 2.60 & 2.62 & 105.9 & 105.6 & 84.51 & 93.21 & 397.75 & 323.00 \\
\hline $35 \mathrm{~kg} / \mathrm{fed}$ & 1283.7 & 1267.6 & 4.15 & 3.92 & 107.8 & 107.2 & 86.74 & 95.36 & 448.50 & 391.91 \\
\hline $70 \mathrm{~kg} / \mathrm{fed}$ & 1461.0 & 1445.9 & 4.77 & 4.25 & 110.0 & 109.5 & 89.27 & 97.22 & 479.50 & 431.16 \\
\hline LSD 0.05 & 0.37 & 0.46 & 0.20 & 0.08 & 0.24 & 0.27 & 1.28 & 0.61 & 0.55 & 0.28 \\
\hline \multicolumn{11}{|l|}{ K levels } \\
\hline $0 \mathrm{~kg} / \mathrm{fed}$ & 1180.6 & 1194.7 & 3.34 & 3.11 & 107.4 & 106.9 & 84.52 & 93.50 & 414.00 & 353.41 \\
\hline $24 \mathrm{~kg} / \mathrm{fed}$ & 1272.1 & 1252.6 & 3.89 & 3.72 & 107.9 & 107.5 & 86.38 & 95.77 & 446.66 & 386.58 \\
\hline $48 \mathrm{~kg} / \mathrm{fed}$ & 1337.3 & 1323.9 & 4.29 & 3.94 & 108.7 & 107.9 & 89.62 & 96.75 & 465.33 & 406.08 \\
\hline LSD 0.05 & 0.21 & 0.20 & 0.11 & 0.08 & 0.26 & 0.21 & 1.02 & 0.60 & 0.46 & 0.27 \\
\hline \multicolumn{11}{|l|}{ Interactions } \\
\hline $\mathrm{G} \times \mathrm{N}$ & $\star *$ & ** & ** & $\star *$ & ** & $* *$ & NS & NS & ${ }^{* *}$ & * \\
\hline GxK & ** & ** & $\star *$ & ** & * & $\star \star$ & NS & $* *$ & NS & NS \\
\hline $\mathrm{NxK}$ & ** & ** & ** & $\star *$ & NS & NS & NS & NS & $* *$ & NS \\
\hline GxNxK & NS & ** & ** & ** & * & $\star *$ & NS & NS & NS & ${ }^{* *}$ \\
\hline
\end{tabular}

\section{Nitrogen rates:}

Data arranged in Tables 2,3 and4 indicated that nitrogen level had positive and significant effect on all the studied traits regarding the current study it was obviously that increasing nitrogen level up to $70 \mathrm{~kg} \mathrm{~N} / \mathrm{fed}$ significantly magnified the growth yield and yield components of rice. Generally, the Lowest values of leaf area index, plant height, mater, number of tillers $/ \mathrm{m}^{2}$, number of panicles $/ \mathrm{m}^{2}$, number of filled grains / panicle , panicle weight, 1000- grain weight, grain yield and straw yield in the first and the second season were obtained when nitrogen fertilizer was not applied (zero nitrogen level). On contrary, the highest values of these traits were produced when the high nitrogen level of $70 \mathrm{~kg} \mathrm{~N} / \mathrm{fed}$ was added for the rice crop in both seasons. The enhancing grain yield of rice resulting from 
increasing nitrogen level might be due to increasing growth photosynthetic rate, net assimilation rate, yield components such as panicles number and number of filled grains / panicle. The findings are in complete conformity with those obtained by El-Shayeb (2003), Gautam et al. (2004), Ebaid(2006), Zayed et al.(2006b) , and Bahmanyar. and Soodaee (2010).

Table 3: Number of panicle $/ \mathrm{m}^{2}$, panicle length $(\mathrm{cm})$, panicle weight $(\mathrm{g})$, number of filled grains/panicle and number of unfilled grains/panicle of some rice genotypes as affected by nitrogen and potassium rates level under saline soils during during2006and2007

\begin{tabular}{|c|c|c|c|c|c|c|c|c|c|c|}
\hline \multirow{2}{*}{\begin{tabular}{|l|} 
Characters \\
treatments \\
Genotypes \\
\end{tabular}} & \multicolumn{2}{|c|}{ Panicles/ $\mathrm{m}^{2}$} & \multicolumn{2}{|c|}{$\begin{array}{l}\text { panicle } \\
\text { length }\end{array}$} & \multicolumn{2}{|c|}{ Panicle weight } & \multicolumn{2}{|c|}{$\begin{array}{c}\text { Filled grains/ } \\
\text { panicle }\end{array}$} & \multicolumn{2}{|c|}{$\begin{array}{l}\text { Unfilled grains } \\
\text { /panicle }\end{array}$} \\
\hline & 2006 & 2007 & 2006 & 2007 & 2006 & 2007 & 2006 & 2007 & 2006 & 2007 \\
\hline Hybrid 1 & 415.97 & 359.96 & 21.94 & 21.60 & 2.68 & 2.79 & 128.28 & 129.55 & 10.83 & 12.02 \\
\hline Hybrid2 & 362.38 & 349.66 & 21.74 & 21.38 & 2.68 & 2.59 & 123.85 & \begin{tabular}{|l|}
124.52 \\
\end{tabular} & 16.83 & 18.33 \\
\hline Giza178 & 308.87 & 306.24 & 21.29 & 21.07 & 2.64 & 2.31 & 124.49 & 122.79 & 09.55 & 11.05 \\
\hline LSD0.05 & 10.80 & 80.00 & 0.32 & 0.45 & 0.02 & 0.05 & NS & 2.45 & 1.16 & 1.76 \\
\hline \multicolumn{11}{|l|}{$\mathrm{N}$ levels } \\
\hline $0 \mathrm{~kg} / \mathrm{fed}$ & 333.75 & 288.25 & 21.18 & 20.25 & 2.39 & 2.07 & 119.67 & 144.63 & 21.22 & 22.27 \\
\hline $35 \mathrm{~kg} / \mathrm{fed}$ & 395.55 & 342.83 & 21.65 & 21.63 & 2.66 & 2.59 & 125.43 & 126.80 & 10.58 & 11.83 \\
\hline $70 \mathrm{~kg} / \mathrm{fed}$ & 439.00 & 384.83 & 22.15 & 22.17 & 2.97 & 3.03 & 127.52 & 135.43 & 05.41 & 07.30 \\
\hline LSD0.05 & 13.50 & 06.00 & 0.26 & 0.29 & 0.03 & 0.03 & 4.02 & 1.74 & 0.76 & 0.80 \\
\hline \multicolumn{11}{|l|}{ K levels } \\
\hline $0 \mathrm{~kg} / \mathrm{fed}$ & 359.08 & 311.83 & 21.02 & 21.39 & 2.46 & 2.33 & 116.81 & 117.66 & 15.11 & 17.47 \\
\hline $24 \mathrm{~kg} / \mathrm{fed}$ & 385.00 & 341.83 & 21.80 & 21.39 & 2.70 & 2.58 & 123.47 & \begin{tabular}{|l|}
126.64 \\
\end{tabular} & 12.03 & 13.47 \\
\hline $48 \mathrm{~kg} / \mathrm{fed}$ & 415.16 & 362.25 & 22.16 & 21.84 & 2.85 & 2.78 & 132.58 & 132.56 & 09.47 & 10.47 \\
\hline LSD0.05 & 08.80 & 06.30 & 0.10 & 0.21 & 0.03 & 0.03 & 3.55 & 1.80 & 0.85 & 0.47 \\
\hline \multicolumn{11}{|c|}{ Interactions } \\
\hline GxN & NS & ${ }^{*}$ & NS & ** & ** & $* *$ & ** & ** & ** & ** \\
\hline GxK & ** & NS & ** & NS & ** & ** & $\star *$ & $\star *$ & ** & ** \\
\hline NxK & NS & ${ }^{* *}$ & $* *$ & NS & $* *$ & ** & NS & NS & ** & $\star *$ \\
\hline GxNxK & NS & NS & NS & NS & NS & NS & NS & NS & NS & NS \\
\hline
\end{tabular}

\section{Potassium levels:}

General speaking, the potassium fertilizer had a fruitful effects on the rice crop involving growth, yield, yield components and growth in both seasons. As presented in Tables 2,3 \&4 increasing potassium fertilizer up to $48 \mathrm{~kg} \mathrm{~K} 20 / \mathrm{fed}$ significantly increased the majority of the studied traits. Rice grain yield and yield components significantly responded to potassium application up to $48 \mathrm{Kg} / \mathrm{fed}$ (Table4). On the other hand, the lowest values of leaf area index, plant height, panicles number $/ \mathrm{m}^{2}$, panicle weight, number of filled grains / panicle, 1000- grain weight, grain yield, straw yield, were observed when no potassium was applied i.e. zero potassium level, while the highest values were found when the high potassium level of $48 \mathrm{~kg} \mathrm{k} \mathrm{k}_{2} \mathrm{O} / \mathrm{fed}$ was applied. Potassium fertilizer didn't affect the harvest index. It is mentioning here, that potassium fertilizer improved both source and sink of rice leading to high grain yield potassium fertilizer encouraged the photosynthesis thesis process and produced large leaf area involving leaf area index which contributes to a great part in the grain filling. In addition, potassium fertilizer 
Hagrus, A. M. et al.

enhanced the reserve of assimilates to sink resulting in more filled grains / panicle, lower unfilled grains/ panicle and more panicles number $/ \mathrm{m}^{2}$. All above-mentioned effects enable potassium to produce considerable gain yield under saline soils.

Table (4):1000-grain weight (g), grain and straw yields (ton/fed) and harvest index of some rice genotypes as affected by nitrogen and potassium levels under saline soil during 2006and2007seasons.

\begin{tabular}{|c|c|c|c|c|c|c|c|c|}
\hline \multirow{2}{*}{\begin{tabular}{|l|} 
Characters \\
Treatments \\
Genotypes \\
\end{tabular}} & \multicolumn{2}{|c|}{$\begin{array}{l}\text { 1000-grain } \\
\text { weight }\end{array}$} & \multicolumn{2}{|c|}{ grain yield } & \multicolumn{2}{|c|}{ Straw yield } & \multicolumn{2}{|c|}{ Harvest index } \\
\hline & 2006 & 2007 & 2006 & 2007 & 2006 & 2007 & 2006 & 2007 \\
\hline Hybrid 1 & 22.68 & 23.76 & 2.42 & 2.67 & 2.19 & 2.56 & 42.17 & 42.71 \\
\hline Hybrid2 & 22.68 & 23.67 & 2.35 & 2.46 & 2.99 & 3.32 & 42.93 & 41.60 \\
\hline Giza178 & 21.07 & 21.56 & 2.29 & 2.14 & 2.76 & 3.32 & 40.94 & 38.68 \\
\hline LSD0.05 & 0.04 & 0.11 & 0.09 & 0.12 & 0.14 & 0.15 & NS & 2.45 \\
\hline \multicolumn{9}{|l|}{$\mathrm{N}$ levels } \\
\hline $0 \mathrm{~kg} / \mathrm{fed}$ & 20.60 & 21.38 & 1.80 & 2.07 & 2.66 & 3.09 & 40.14 & 39.29 \\
\hline $35 \mathrm{~kg} / \mathrm{fed}$ & 22.39 & 23.33 & 2.81 & 1.42 & 2.91 & 3.34 & 43.63 & 41.61 \\
\hline $70 \mathrm{~kg} / \mathrm{fed}$ & 23.50 & 24.38 & 2.69 & 2.79 & 3.36 & 3.87 & 42.61 & 41.55 \\
\hline LSD0.05 & 0.02 & 0.04 & 0.11 & 0.13 & 0.18 & 0.17 & 4.02 & 1.74 \\
\hline \multicolumn{9}{|l|}{ K levels } \\
\hline $0 \mathrm{~kg} /$ fed & 21.36 & 22.49 & 1.96 & 2.18 & 2.65 & 3.22 & 41.69 & 40.17 \\
\hline $24 \mathrm{~kg} / \mathrm{fed}$ & 22.39 & 23.14 & 2.36 & 2.50 & 3.06 & 3.44 & 42.61 & 41.29 \\
\hline $48 \mathrm{~kg} / \mathrm{fed}$ & 22.84 & 23.47 & 2.65 & 2.58 & 3.23 & 3.64 & 42.07 & 41.00 \\
\hline LSD0.05 & 0.03 & 0.06 & 0.09 & 0.09 & 0.13 & 0.16 & NS & NS \\
\hline \multicolumn{9}{|l|}{ Interactions } \\
\hline $\mathrm{G} \times \mathrm{N}$ & ** & ** & ** & ** & NS & NS & NS & NS \\
\hline GxK & NS & NS & NS & NS & NS & NS & NS & NS \\
\hline $\mathrm{NxK}$ & NS & NS & $* *$ & $\star *$ & NS & NS & NS & NS \\
\hline GxNxK & NS & NS & NS & NS & NS & NS & NS & NS \\
\hline
\end{tabular}

These findings are in a harmony with those reported by Nour et al. (1997) Ebaid and Ghanem (2001) and as well as Zayed et al. (2007) and Bahmanyar and Soodaee (2010).

\section{The interaction effect:}

The interaction between rice genotypes and nitrogen levels had significant effect on dry matter, IAI, number of tillers/m2 in both seasons, number of panicles/m2 in 2007 season only, number of filed grains /panicle, number of unfilled grains/panicle and panicle weight in both seasons. The rest of measured traits including grain yield didn't response significantly to the interaction between rice genotypes and nitrogen levels in both seasons. The current interaction showed the superiority of the combination of EHR1 and 70 $\mathrm{kg} \mathrm{N} / \mathrm{fed}$ under current conditions (Table 5\&6).

The interaction between rice genotypes and potassium levels had significant effect on dry matter, LAl, number of tillers $/ \mathrm{m} 2$, number of panicles/m2 in 2007 seasons, number of filed grains /panicle, number of unfilled grains/panicle and panicle weight in both seasons. The rest of measured traits including grain yield didn't response significantly to the interaction between genotypes and potassium levels in both seasons. The 
current interaction showed the superiority of the combination of EHR1 and 48 $\mathrm{kg} \mathrm{k}_{2} \mathrm{O} / \mathrm{fed}$ under current conditions (Table 7\&8).

Table 5 :LAl, dry matter/ $\mathrm{m}^{2}$, number of tillers/ $\mathrm{m}^{2}$, number of panicles/ $\mathrm{m}^{2}$ as affected by the interaction between rice genotypes and nitrogen levels during 2006and2007seasons.

\begin{tabular}{|c|c|c|c|c|c|c|c|c|}
\hline \multirow{2}{*}{ Genotypes } & \multirow{2}{*}{$\begin{array}{c}\mathbf{N} \\
\text { Level } \\
\text { skg } \\
\text { N/fed }\end{array}$} & \multicolumn{2}{|c|}{ dry matter/ m² } & \multicolumn{2}{|c|}{ LAl } & \multicolumn{2}{|c|}{ number of tillers $/ \mathrm{m}^{2}$} & \multirow{2}{*}{$\begin{array}{c}\begin{array}{c}\text { number of } \\
\text { panicles/ } \mathrm{m}^{2}\end{array} \\
2007 \\
\end{array}$} \\
\hline & & 2006 & 2007 & 2006 & 2007 & 2006 & 2007 & \\
\hline \multirow[t]{3}{*}{ SK2034H } & 0 & 1130.7 & 1115.5 & 2.58 & 2.65 & 420.91 & 335.33 & 297.91 \\
\hline & 35 & 1243.4 & 1244.0 & 4.43 & 4.56 & 473.83 & 419.58 & 372.83 \\
\hline & 70 & 1480.1 & 1468.3 & 5.05 & 4.95 & 523.41 & 452.83 & 409.16 \\
\hline \multirow[t]{3}{*}{ SK2046H } & 0 & 987.4 & 935.8 & 2.57 & 2.65 & 417.75 & 347.00 & 309.66 \\
\hline & 35 & 1351.1 & 1345.8 & 4.33 & 4.39 & 429.83 & 395.50 & 338.00 \\
\hline & 70 & 1495.2 & 1481.3 & 4.56 & 4.55 & 447.00 & 445.91 & 401.33 \\
\hline \multirow[t]{3}{*}{ Giza 178} & 0 & 1015.5 & 992.2 & 2.64 & 2.55 & 354.33 & 286.58 & 257.25 \\
\hline & 35 & 1226.7 & 1213.5 & 3.67 & 2.83 & 442.75 & 360.83 & 317.41 \\
\hline & 70 & 1410.2 & 1388.0 & 4.70 & 3.22 & 476.91 & 394.75 & 344.08 \\
\hline LSD0.05 & & 65.13 & 78.98 & 0.37 & 0.14 & 0.90 & 0.48 & 0.44 \\
\hline
\end{tabular}

Table 6:Filled and unfilled grains/panicle, panicle weight(g)and grain yield(ton/fed) as affected by the interaction between rice genotypes and nitrogen levels during 2006and2007seasons

\begin{tabular}{|c|c|c|c|c|c|c|c|c|c|}
\hline \multirow[t]{2}{*}{ genotypes } & \multirow{2}{*}{\begin{tabular}{|c|}
$\mathbf{N}$ \\
levels \\
Kg N/fed \\
\end{tabular}} & \multicolumn{2}{|c|}{$\begin{array}{c}\text { Filled } \\
\text { grains/panicle }\end{array}$} & \multicolumn{2}{|c|}{$\begin{array}{c}\text { unfilled } \\
\text { grains/panicle }\end{array}$} & \multicolumn{2}{|c|}{$\begin{array}{l}\text { Panicle } \\
\text { weight }\end{array}$} & \multicolumn{2}{|c|}{$\begin{array}{l}\text { Grain yield } \\
\text { ton/fed) ( }\end{array}$} \\
\hline & & 2006 & 2007 & 2006 & 2007 & 2006 & 2007 & 2006 & 2007 \\
\hline \multirow[t]{3}{*}{ SK2034H } & 0 & 116.83 & 119.08 & 19.75 & 20.58 & 2.20 & 2.19 & 2.08 & 2.12 \\
\hline & 35 & 128.58 & 129.83 & 8.75 & 10.25 & 2.62 & 2.88 & 2.36 & 2.38 \\
\hline & 70 & 139.45 & 139.76 & 4.00 & 5.25 & 3.24 & 3.34 & 2.73 & 2.78 \\
\hline \multirow[t]{3}{*}{ SK2046H } & 0 & 115.80 & 117.81 & 27.33 & 28.91 & 2.60 & 2.23 & 1.62 & 1.69 \\
\hline & 35 & 125.98 & 126.56 & 12.25 & 16.25 & 2.64 & 2.54 & 1.95 & 2.06 \\
\hline & 70 & 129.89 & 129.20 & 7.91 & 9.83 & 2.81 & 2.95 & 2.24 & 2.27 \\
\hline \multirow[t]{3}{*}{ Giza 178} & 0 & 110.18 & 107.02 & 16.58 & 17.33 & 2.36 & 1.81 & 1.48 & 1.50 \\
\hline & 35 & 123.44 & 124.02 & 7.75 & 9.00 & 2.71 & 2.35 & 1.82 & 1.87 \\
\hline & 70 & 139.87 & 137.33 & 4.33 & 6.83 & 2.86 & 2.97 & 2.07 & 2.09 \\
\hline LSD0.05 & & 2.57 & 3.21 & 1.47 & 1.93 & 0.05 & 0.06 & 0.013 & 0.016 \\
\hline
\end{tabular}

The interaction between nitrogen and potassium levels had significant effect on dry matter, LAl, number of panicles/m2 in 2006 season only, number of unfilled grains/panicle and panicle weight in both seasons. The rest of measured traits including grain yield didn't response significantly to the interaction between nitrogen and potassium levels in both seasons. The current interaction showed the superiority of the combination of $60 \mathrm{~kg} \mathrm{~N} / \mathrm{fed}$ and $48 \mathrm{~kg} \mathrm{k} 2 \mathrm{O} / \mathrm{fed}$ under current conditions (Table9\&10). 
Hagrus, A. M. et al.

Table7: dry matter/ $\mathbf{m}^{2}$,LAl, number of tillers $/ \mathbf{m}^{2}$ and number of panicle/ $\mathrm{m}^{2}$ as affected by the interaction between rice genotypes and potassium levels during 2006and2007seasons

\begin{tabular}{|c|c|c|c|c|c|c|c|}
\hline \multirow[t]{2}{*}{ genotypes } & \multirow{2}{*}{$\stackrel{\mathrm{K}}{\text { levels } \mathrm{kg} \mathrm{K} \mathrm{K}_{2} \mathrm{O}}$} & \multicolumn{2}{|c|}{ Filled grains/panicle } & \multicolumn{2}{|c|}{$\begin{array}{c}\text { unfilled } \\
\text { grains/panicle }\end{array}$} & \multicolumn{2}{|c|}{$\begin{array}{c}\text { Panicle weight } \\
\text { (g) }\end{array}$} \\
\hline & & 2006 & 2007 & 2006 & 2007 & 2006 & 2007 \\
\hline \multirow[t]{3}{*}{ SK2034H } & 0 & 119.56 & 123.45 & 13.91 & 15.58 & 2.49 & 2.61 \\
\hline & 24 & 128.13 & 129.11 & 10.50 & 11.66 & 2.69 & 2.77 \\
\hline & 48 & 137.15 & 136.32 & 8.08 & 8.83 & 2.88 & 3.00 \\
\hline \multirow[t]{3}{*}{ SK2046H } & 0 & 113.83 & 113.74 & 21.33 & 23.16 & 2.50 & 2.32 \\
\hline & 24 & 125.76 & 126.67 & 16.08 & 17.50 & 2.69 & 2.61 \\
\hline & 48 & 132.18 & 133.15 & 13.08 & 14.33 & 2.85 & 2.79 \\
\hline \multirow[t]{3}{*}{ Giza 178} & 0 & 117.03 & 115.99 & 11.91 & 13.66 & 3.39 & 2.06 \\
\hline & 24 & 128.07 & 124.15 & 9.50 & 12.25 & 2.71 & 2.36 \\
\hline & 48 & 128.39 & 128.22 & 7.25 & 8.25 & 2.38 & 2.54 \\
\hline LSD0.05 & & 5.04 & 3.30 & 1.56 & 9.90 & 0.04 & 0.06 \\
\hline
\end{tabular}

Table 8: Filled and unfilled grains/panicle, panicle weight $(\mathrm{g})$ and grain yield as affected by the interaction between rice genotypes and potassium levels in2006and2007seasons

\begin{tabular}{|c|c|c|c|c|c|c|}
\hline \multirow[b]{2}{*}{ Genotypes } & \multirow{2}{*}{$\begin{array}{c}\mathrm{K} \\
\text { levels } \\
\mathrm{kgk}_{2} \mathrm{O} / \\
\text { fed }\end{array}$} & \multicolumn{2}{|c|}{ dry matter/ m² } & \multirow{2}{*}{$\begin{array}{r}\text { LAl } \\
2006\end{array}$} & \multicolumn{2}{|c|}{ number of panicle $/ \mathrm{m}^{2}$} \\
\hline & & 2006 & 2007 & & 2007 & 2006 \\
\hline \multirow[t]{3}{*}{ SK2034H } & 0 & 1222.6 & 1188.2 & 3.22 & 3.29 & 393.53 \\
\hline & 24 & 1305.9 & 1285.6 & 4.27 & 4.30 & 410.91 \\
\hline & 48 & 1355.7 & 1348.4 & 4.57 & 4.57 & 443.41 \\
\hline \multirow[t]{3}{*}{ SK2046H } & 0 & 1214.0 & 1186.6 & 3.48 & 3.56 & 327.83 \\
\hline & 24 & 1280.7 & 1256.5 & 3.72 & 3.50 & 356.83 \\
\hline & 48 & 1338.7 & 1319.3 & 4.27 & 4.14 & 402.50 \\
\hline \multirow[t]{3}{*}{ Giza 178} & 0 & 1104.3 & 1074.3 & 3.31 & 2.48 & 355.75 \\
\hline & 24 & 1229.6 & 1215.7 & 3.69 & 2.98 & 387.41 \\
\hline & 48 & 1318.0 & 1303.9 & 4.02 & 3.12 & 399.75 \\
\hline LSD0.05 & & 51.16 & 57.12 & 0.28 & 0.13 & 15.50 \\
\hline
\end{tabular}

Table 9: dry matter $/ \mathrm{m}^{2}$ LAI number of tiller $/ \mathrm{m}^{2}$ and number of panicle/ $\mathrm{m}^{2}$ as affected by the interaction between nitrogen and potassium levels during 2006and2007seasons

\begin{tabular}{|c|c|c|c|c|c|c|}
\hline \multirow{2}{*}{\begin{tabular}{|c|c|}
$\mathbf{N}$ \\
$\begin{array}{c}\text { Level kg } \mathbf{N} \\
\text { /fed }\end{array}$ \\
\end{tabular}} & \multirow{2}{*}{$\begin{array}{c}\mathrm{K} \\
\text { level kg } \\
\mathrm{K}_{2} \mathrm{O} / \mathrm{fed} \\
\end{array}$} & \multicolumn{2}{|c|}{ dry matter/ m $^{2}$} & LAI & \multicolumn{2}{|c|}{ number of panicle/m² } \\
\hline & & 2006 & 2007 & 2006 & 2007 & $200 v$ \\
\hline \multirow[t]{3}{*}{0} & 0 & 1012.5 & 951.0 & 2.32 & 2.40 & 256.00 \\
\hline & 24 & 1033.0 & 1005.0 & 2.49 & 2.60 & 289.50 \\
\hline & 48 & 1087.6 & 1082.0 & 2.99 & 3.84 & 319.25 \\
\hline \multirow[t]{3}{*}{35} & 0 & 1182.8 & 1167.0 & 3.52 & 3.25 & 320.75 \\
\hline & 24 & 1315.8 & 1293.8 & 4.30 & 4.19 & 347.25 \\
\hline & 48 & 1352.4 & 1341.9 & 4.62 & 4.32 & 360.50 \\
\hline \multirow[t]{3}{*}{70} & 0 & 1346.4 & 1337.0 & 4.18 & 3.67 & 358.75 \\
\hline & 24 & 1467.5 & 1458.9 & 4.89 & 4.39 & 388.75 \\
\hline & 48 & 1571.6 & 1547.7 & 5.25 & 4.67 & 407.00 \\
\hline LSD0.05 & & 47.35 & 53.96 & 0.26 & 0.15 & 10.50 \\
\hline
\end{tabular}


Table10:Filled and unfilled grain/panicle panicle weight(g)and grain yield as affected by the interaction between nitrogen and potassium levels during2006and2007seasons

\begin{tabular}{|c|c|c|c|c|c|c|c|}
\hline \multirow{2}{*}{\begin{tabular}{|c|}
$\mathbf{N}$ \\
$\begin{array}{c}\text { levels kg N } \\
\text { /fed }\end{array}$ \\
\end{tabular}} & \multirow{2}{*}{$\begin{array}{c}\mathrm{K} \\
\text { levels kg } \\
\mathrm{K}_{2} \mathrm{O} / \mathrm{fed} \\
\end{array}$} & \multicolumn{2}{|c|}{$\begin{array}{c}\text { Unfilled } \\
\text { grains/panicle }\end{array}$} & \multicolumn{2}{|c|}{$\begin{array}{c}\text { Panicle weight } \\
\text { (g) }\end{array}$} & \multicolumn{2}{|c|}{ Grain yield ton/fed) } \\
\hline & & 2006 & 2007 & 2006 & 2007 & 2006 & 2007 \\
\hline \multirow[t]{3}{*}{0} & 0 & 26.75 & 28.33 & 2.18 & 1.80 & 1.64 & 1.70 \\
\hline & 24 & 20.42 & 21.58 & 2.43 & 2.12 & 1.73 & 1.78 \\
\hline & 48 & 16.50 & 16.92 & 2.56 & 2.30 & 1.81 & 1.83 \\
\hline \multirow[t]{3}{*}{35} & 0 & 21.58 & 13.92 & 2.41 & 2.43 & 1.97 & 2.00 \\
\hline & 24 & 10.75 & 12.08 & 2.70 & 2.60 & 2.02 & 2.12 \\
\hline & 48 & 8.42 & 9.50 & 2.87 & 2.75 & 2.15 & 2.19 \\
\hline \multirow[t]{3}{*}{70} & 0 & 7.83 & 10.17 & 2.81 & 2.77 & 2.28 & 2.33 \\
\hline & 24 & 4.92 & 6.75 & 2.97 & 3.03 & 2.33 & 2.38 \\
\hline & 48 & 3.50 & 5.00 & 3.14 & 3.29 & 2.43 & 2.44 \\
\hline LSD0.05 & & 1.40 & 1.27 & 0.44 & 0.05 & 0.029 & 0.016 \\
\hline
\end{tabular}

The tri-interaction among rice varieties, nitrogen and potassium levels had insignificant effect on dry matter, IAI, number of tillers $/ \mathrm{m} 2$, number of panicles/m2 in 2007 seasons, number of unfilled grains/panicle and panicle weight in both seasons. The tri- interaction showed the superiority of the combination of EHR1, $70 \mathrm{~kg} \mathrm{~N} / \mathrm{fed}$ and $48 \mathrm{~kg} \mathrm{k} 2 \mathrm{O} / \mathrm{fed}$ under current conditions in rice grain yield in both seasons. All rice varieties growing under saline soil need to potassium fertilizer by the same amount of $48 \mathrm{~kg} \mathrm{k} \mathrm{k}_{2} \mathrm{O}$ /fed(Table 11). It could be concluded that EHR1 had to be fertilized by70 kg $\mathrm{N} / \mathrm{fed}$ and $48 \mathrm{~kg} \mathrm{k}_{2} \mathrm{O} / \mathrm{fed}$ under current saline conditions for producing considerable grain yield.

Table 11: grain yield ( $t / f e d)$ of some rice genotypes as affected by the tri-interaction during 2006 and 2007 seasons.

\begin{tabular}{|c|c|c|c|c|c|c|c|c|c|}
\hline \multirow{4}{*}{ Genotypes } & \multirow{4}{*}{$\begin{array}{l}\mathrm{N} \text { levels } \\
\text { kg fed }^{-1} \text {. }\end{array}$} & \multicolumn{8}{|c|}{ Grain yield $t / f e d$} \\
\hline & & \multirow{2}{*}{\multicolumn{4}{|c|}{$\begin{array}{c}2006 \\
\text { K level kg k } \mathbf{k}_{2} \mathrm{O} \text { fed }\end{array}$}} & \multicolumn{4}{|c|}{2007} \\
\hline & & & & & & & evel k & ${ }_{2} \mathrm{O} \mathrm{fe}$ & \\
\hline & & 0 & 24 & 48 & mean & 0 & 24 & 48 & mean \\
\hline \multirow{4}{*}{ SK2034H } & 0 & 1.88 & 2.01 & 2.25 & 2.04 & 2.17 & 2.32 & 2.4 & 2.31 \\
\hline & 35 & 2.13 & 2.45 & 2.57 & 2.38 & 2.37 & 2.61 & 2.81 & 2.59 \\
\hline & 70 & 2.40 & 2.90 & 3.18 & 2.83 & 2.71 & 3.20 & 3.37 & 3.09 \\
\hline & mean & 2.13 & 2.46 & 2.67 & 2.42 & 2.42 & 2.71 & 2.87 & 2.67 \\
\hline \multirow{4}{*}{ SK2046H } & 0 & 1.58 & 1.84 & 1.93 & 1.78 & 1.77 & 2.10 & 2.17 & 2.01 \\
\hline & 35 & 2.02 & 2.72 & 3.31 & 2.68 & 2.22 & 2.67 & 2.92 & 2.60 \\
\hline & 70 & 2.18 & 2.76 & 2.85 & 2.59 & 2.37 & 2.90 & 3.02 & 2.76 \\
\hline & mean & 1.92 & 2.44 & 2.70 & 2.35 & 2.12 & 2.55 & 2.70 & 2.46 \\
\hline \multirow{4}{*}{ Giza178 } & 0 & 1.52 & 1.63 & 1.59 & 1.58 & 1.80 & 1.82 & 2.02 & 1.88 \\
\hline & 35 & 1.72 & 2.28 & 2.55 & 2.18 & 1.88 & 2.40 & 2.45 & 2.24 \\
\hline & 70 & 2.26 & 2.68 & 3.03 & 2.66 & 2.37 & 2.55 & 2.65 & 2.52 \\
\hline & mean & 1.84 & 2.20 & 2.39 & 2.14 & 2.02 & 2.25 & 2.37 & 2.21 \\
\hline LSD 0.05 & & \multicolumn{4}{|c|}{0.029} & \multicolumn{4}{|c|}{0.026} \\
\hline
\end{tabular}




\section{REFERENCES}

Abou-Khalifa, A. A.(2005). Physiological behavior of some rice cultivars under different sowing dates and seedling ages. The $11^{\text {th }}$ conference of agronomy, Agron. Dept.Fac.Agric.,Assuit Univ.,Nov.15-16 p:315-327

Bahmanyar M. A. and S. Soodaee (2010). Influence of nitrogen and potassium top dressing on yield and yield components as well as their accumulations in rice (Oryza sativa). African Journal of Biotechnology Vol. 9(18):2648-2653.

Dunn D. and G.Stevens. (2005). Rice potassium nutrition research progress. Better crops with plant food . 89 (1) : $15-17$.

Ebaid, R.A. and S.A. Ghanem (2001). Effects of nitrogenous and potash fertilizers on the productivity of Sakha 101 rice cultivar. J.Agric. Sci. Mansoura univ. 26 (4) : $1833-1840$.

El - Hawary, A; M.A.; A.A.Abd El - Rahman and G.A. Dowiadar (2009). Effect of irrigation water deficit and potassium fertilization on some rice varieties. J.Agric Sci. Mansoura Univ., 34 (7) : 8081 - 8092.

El-Shayeb,O.M. (2003). Effect of hill spacings, nitrogen levels and harvest date on rice productivity and grain quality. M.SC. thesis fac. of Agric., Agron. Dept., Mansoura univ., Egypt.

Ghanem, S.A (2002). Mineral nutrition (potassium). Rice in Egypt Rice Research and Training center, Sakha, Kafr - El Sheikh page 111.

Gautam.A.K (2004) Effect of nitrogen level and spacing on productivity and quality of inbred and hybrid aromatic rice PHD. Thesis, Division of Agronomy, Indian Agric., Res.Instit., New Delhi, India.

Gomez,K .A. and A.A Gomez (1984). Statistical procedures for agricultural research. $2^{\text {nd }}$ Ed. John Wiley and Sons, New York, USA.

Lin X. Q., D. F. Zhu, H. Z. Chen, S. H. Cheng and N. Up off (2009):Effect of plant density and nitrogen fertilizer rates on grain yield and nitrogen uptake of hybrid rice (Oryza sativa L.). Journal of Agricultural Biotechnology and Sustainable Development Vol. 1(2) pp. 044-053.

Nour, M.A.; A.E. Abd El-Wahab;A.A. El-Kady and R.A. Ebaid, (1997). Productivity of some rice varieties under different irrigation intervals and potassium fertilizer. Egypt . J. of App.Sci. 12 (6) : 137 - 154 .

Shahzada M. M. M; Sarfraz and M. Hafeez (2007). Response of rice advance Line PB-95 to potassium application in Saline-sodic Soil. Pakistan J. of Biological Sci., 10:2935-2939.

Zayed, B.A.; S.M. Shehata ;A.A.Abd El Rahaman and A.E. Draz (2005). Cell membrane stability, physiology rate and associated traits as an indictor of salinity tolerance in some rice cultivars (Oryza sativa L.,). Egypt.J.of Agric.,Res.,82(5B):411-423

Zayed, B.A.; A.E.Abd El Wahab ;A.O.Basttawisi ;A.M.Hagras and H.F.W.ElMowafi (2006a). Physiology response of some hybrid and inbred rice varieties to different times of nitrogen application and plant geometry. First Filed Crop Conference, 22-24, Agust .Giza ,Egypt,pp.533-542. 
Zayed B. A.; A.M.El Ekhtyar; A. B. El Abd and M.A. Badawi (2006b). Response of hybrid and inbred rice varieties to various nitrogen levels under saline soil conditions .J.Agric. Sci., Mansoura Univ .,31 (12) :7497 - 7509.

Zayed, B.A.; W.M. Elkhoby; S.M.Shehata and M.H. Ammar (2007). Role of potassium application on the productivity of some inbred and hybrid rice varieties under newly reclaimed saline soils. $8^{\text {th }}$ African crop Sci society Conference,27-31 October, El-Minia, Egypt (1):55-60.

Zayed,B.A.; I.S.El-Rafaee and S.E.M.Sedeek (2010). Response of different rice varieties to phosphorus fertilizer under newly reclaimed saline soil J. Plant Prod. Mansoura Univ., 1(11):1479-1493.

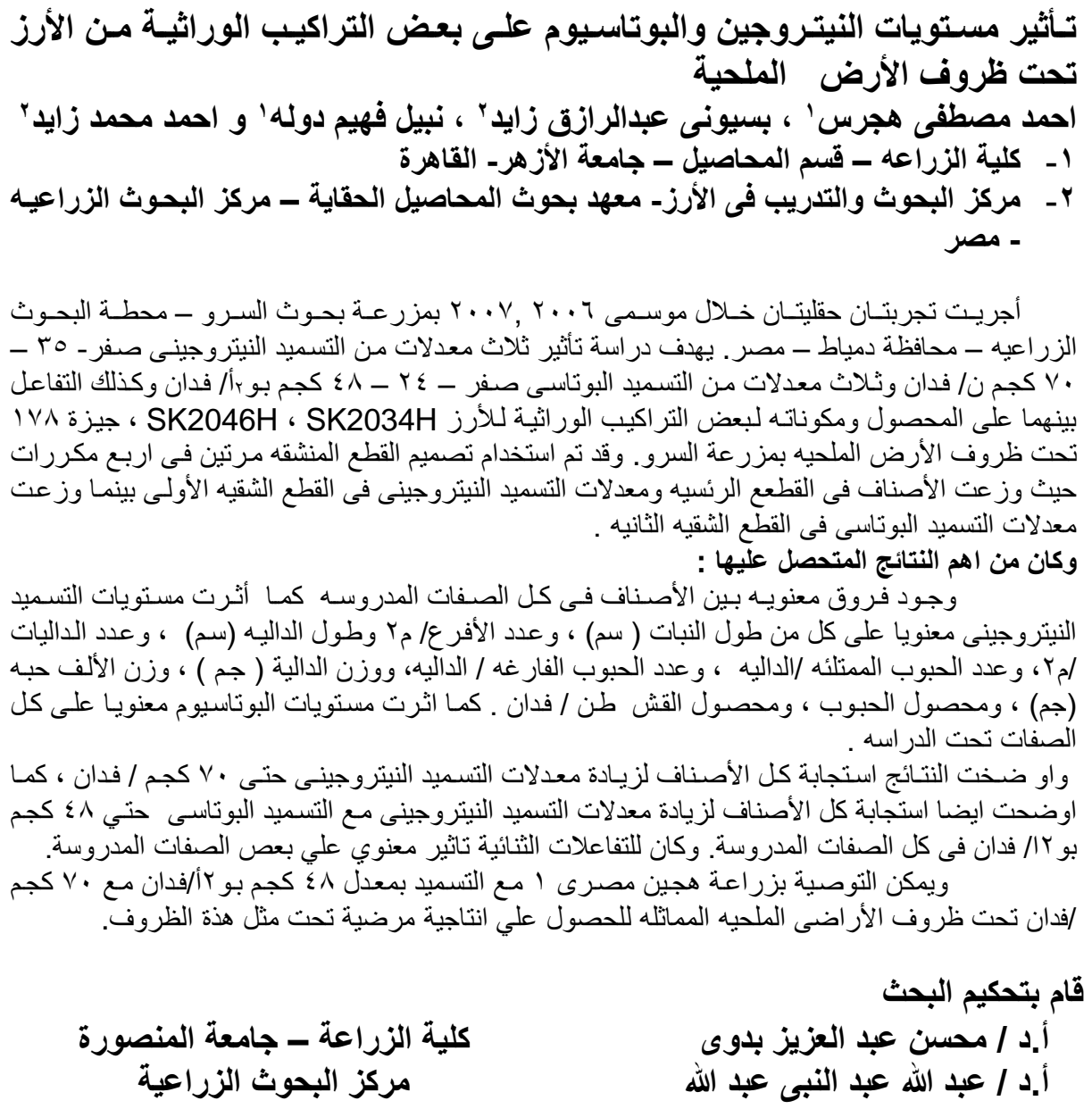

كلية الزراعة - جامعة المنصورة

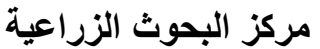

قام بتحكيم البحث

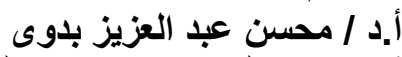

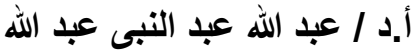

